\title{
Network analysis of the genomic basis of the placebo effect
}

\author{
Rui-Sheng Wang, ${ }^{1}$ Kathryn T. Hall, ${ }^{1,2}$ Franco Giulianini, ${ }^{1,2}$ Dani Passow, ${ }^{3}$ Ted J. Kaptchuk, ${ }^{3}$ \\ and Joseph Loscalzo'
}

'Department of Medicine and 'Division of Preventive Medicine, Brigham and Women's Hospital, Harvard Medical School, Boston, Massachusetts, USA. ${ }^{3}$ Program in Placebo Studies, Beth Israel Deaconess Medical Center, Harvard Medical School, Boston, Massachusetts, USA.

The placebo effect is a phenomenon in which patients who are given an inactive treatment (e.g., inert pill) show a perceived or actual improvement in a medical condition. Placebo effects in clinical trials have been investigated for many years especially because placebo treatments often serve as the control arm of randomized clinical trial designs. Recent observations suggest that placebo effects may be modified by genetics. This observation has given rise to the term "placebome," which refers to a group of genome-related mediators that affect an individual's response to placebo treatments. In this study, we conduct a network analysis of the placebome and identify a placebome module in the comprehensive human interactome using a seed-connector algorithm. The placebome module is significantly enriched with neurotransmitter signaling pathways and brain-specific proteins. We validate the placebome module using a large cohort of the Women's Genome Health Study (WCHS) trial and demonstrate that the placebome module is significantly enriched with genes whose SNPs modify the outcome in the placebo arm of the trial. To gain insights into placebo effects in different diseases and drug treatments, we use a network proximity measure to examine the closeness of the placebome module to different disease modules and drug target modules. The results demonstrate that the network proximity of the placebome module to disease modules in the interactome significantly correlates with the strength of the placebo effect in the corresponding diseases. The proximity of the placebome module to molecular pathways affected by certain drug classes indicates the existence of placebo-drug interactions. This study is helpful for understanding the molecular mechanisms mediating the placebo response, and sets the stage for minimizing its effects in clinical trials and for developing therapeutic strategies that intentionally engage it.

Conflict of interest: The authors have declared that no conflict of interest exists.

Submitted: March 8, 2017

Accepted: May 2, 2017

Published: June 2, 2017

Reference information: JCI Insight. 2017;2(11):e93911. https:// doi.org/10.1172/jci.insight.93911.

\section{Introduction}

A placebo is an inert treatment designed to simulate a biomedical intervention. Despite their lack of active principles, placebos have helped to ease symptoms of illness for centuries (1). Placebos have also been used for many years as control treatments in clinical studies designed to test new drug therapies (2). The placebo effect is a phenomenon in which patients who are given an inactive treatment show a perceived or actual improvement in a medical condition (3). As commonly used, placebo effects are typically beneficial effects in a patient resulting from placebo treatments. Unpleasant effects or frankly adverse events that occur after placebo treatments are denoted nocebo (I will harm, Lat.) effects (4). Recent clinical research has provided compelling evidence that placebo effects have a physiological basis, rather than being a reflection of simple spontaneous remission or natural symptom fluctuations $(5,6)$.

Placebo effects vary widely in patient groups with different diseases and also differ in the same disease groups administered different drug treatments (7), indicating the coexistence of placebo-disease interactions and placebo-drug interactions, respectively. Although it has been consistent as to whether response rates to placebo treatments have remained constant or increased over time $(8,9)$, the placebo response is highly heterogeneous within a patient population. The reasons underlying this variation remain controversial. With advances in genomics, researchers have begun to recognize that placebo responses are a complex phenotype with an evolving physiology that may be modified by an individual's genetic context. We recently proposed the concept of "the placebome," which represents a group of genome-related or derived molecules that 
affects an individual's response to placebo treatments (10). The concept of a placebome raises a series of interesting and challenging questions in basic and clinical research, as well as therapeutics.

A few studies have shown that genetic variation in the brain's neurotransmitter (e.g., endorphin, cannabinoid, dopamine, and opioid) pathways may modify placebo responses $(6,10)$. For example, the catecholO-methyltransferase (COMT) gene, which contains an exonic SNP (rs4680) that can reduce its enzymatic activity, was reported to be a placebo biomarker (11). Genetic variations in COMT can influence the brain's level of the neurotransmitter dopamine and may, thereby, influence the extent of an individual's placebo response. Accumulating evidence supports the potential existence of a group of genes or gene products that mediate placebo responses in individual patients. In the past decade, high-throughput technologies have generated a large amount of genomic data and protein-protein interaction data that facilitate systems biology and network medicine studies (12-14). Such omics data offer unprecedented opportunities to investigate the mechanism of placebo effects in different diseases at the systems level.

Detailed knowledge of the placebome will help us understand how genomics might influence the role of the placebo in patient care and clinical research, and eventually allow researchers to tailor treatments to individuals and more efficiently disentangle drug effects from placebo effects (3). In this study, we aim to connect the placebome with the comprehensive human interactome in order to identify a subnetwork of interacting proteins that mediate the underlying mechanism(s) of placebo effects. The questions we propose to explore include: Is there a distinct placebome module located in a network neighborhood of the human interactome? What are the relationships between the placebome module and different diseases or drug categories at the systems level? Can we predict the strength of placebo effects in patient populations with different diseases? Does such network analysis indicate the existence of placebo-drug interactions? Addressing these questions has great potential to improve our understanding of the mechanisms underlying placebo effects and to guide the development of novel strategies for both clinical trial design and precision medicine-based treatments.

\section{Results}

The placebome module. The first question we explored is whether there is a network neighborhood in the comprehensive human interactome determining the placebome module that may underlie placebo responses. To this end, we ascertained the subnetwork that the placebome seed proteins comprise. As shown in Figure 1A, the subnetwork induced by mapping the placebome seed proteins to the interactome includes 16 proteins and 21 interactions. The size of its largest connected component (LCC) is 6; the diameter of the subnetwork is 1.04 . To assess the significance of the topological properties of this subnetwork, we randomly selected the same number (28) of proteins from the human interactome 1,000 times and determined the properties of the induced subnetworks. As shown in Figure 1, B and C, the subnetwork induced by the placebome seed genes is significantly denser than those induced by random protein sets $\left(P<1.0 \times 10^{-16}\right)$ and the size of its LCC is significantly larger $\left(P=1.7 \times 10^{-10}\right)$. The diameter of the placebome seed protein set, defined as the average distance of each seed protein to its closest proteins in the set (14), is significantly smaller $\left(P=2.8 \times 10^{-23}\right.$, Figure 1D). These results indicate that the subnetwork induced by the placebome seed proteins is not random and has significant, distinct topological properties.

Owing to the incomplete knowledge of the placebome and the human interactome, some placebome seed proteins are isolated and not connected to other seed proteins to form a larger module. Therefore, we developed an algorithm, denoted the Seed-Connector algorithm (see Methods), to connect the placebome seed genes by adding as few extra nodes (seed connectors) as possible. The network induced by the placebome seed proteins and the seed connectors is shown in Figure 2. This network has 54 nodes (including 26 seed connectors) and 108 interactions, with 27 of the 28 placebome seed proteins connected in the resulting subnetwork. We refer to this subnetwork of the interactome as the placebome module. Compared with the subnetwork induced by a random protein set of the same size, the placebome module is significantly denser and has a significantly larger LCC (Supplemental Figure 1; supplemental material available online with this article; https://doi.org/10.1172/jci.insight.93911DS1). In addition, the placebome module has a significantly smaller diameter and average shortest path length (Supplemental Figure 1), indicating that the placebome module is a network cluster in the human interactome.

Among the 26 seed connectors (predicted placebome proteins), some are brain-specific proteins or proteins from neurotransmitter signaling pathways. For example, OPRD1 (opioid receptor, $\delta 1$ ) is a member of the opioid family of $G$ protein-coupled receptors that also includes $\mu$, $\kappa$, and nociceptin 
A
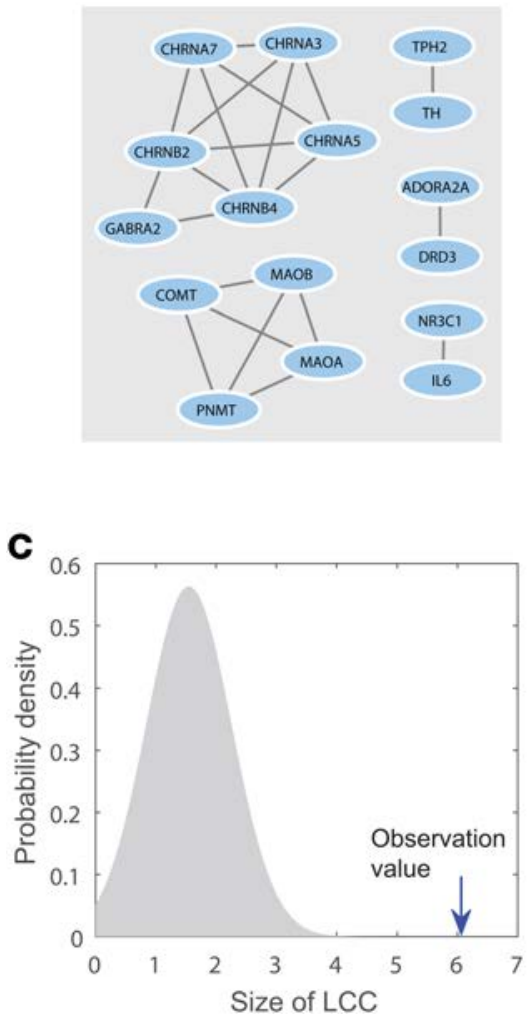

B

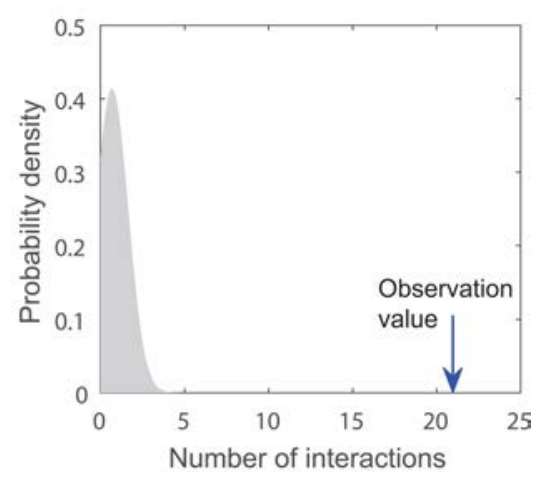

D

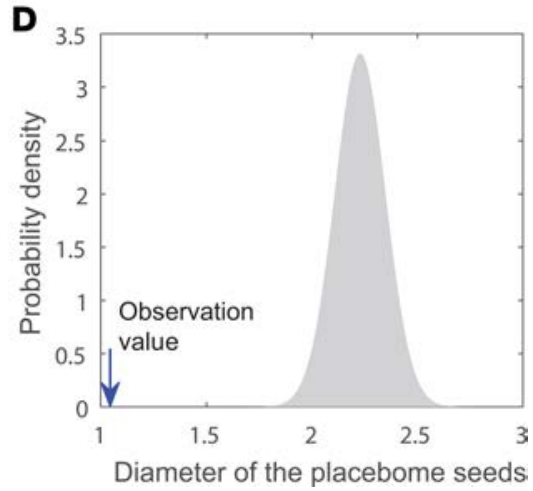

Figure 1. The subnetwork induced by the placebome seed proteins and its topological properties. (A) The subnetwork induced by the placebome seed proteins. (B) The subnetwork has significantly more interactions than random expectation. (C) The subnetwork has a significantly greater largest connected component (LCC) than random expectation. (D) The subnetwork has a significantly smaller diameter than random expectation.

(NOP) receptors and is associated with neurological diseases and disorders (15), including neonatal abstinence syndrome and morphine dependence. STX1A (syntaxin 1A) is a nervous system-specific protein implicated in the docking of synaptic vesicles with the presynaptic plasma membrane. YWHAZ (tyrosine 3-monooxygenase/tryptophan 5-monooxygenase activation protein, $\zeta$ ) is a protein whose mRNA is widely expressed in various gray matter regions of the brain. YWHAG (tyrosine 3-monooxygenase/tryptophan 5 monooxygenase activation protein, $\gamma$ ) is highly expressed in brain, skeletal muscle, and heart. The complete list of the proteins in the placebome module can be found in Supplemental Table 1 (Supplemental File 2).

We next systematically examined whether the placebome module is significantly enriched with tissuespecific proteins in the brain. Tissue-specific expression data were compiled from the Human Protein Atlas (HPA) (16), wherein 1,437 genes are identified as elevated in the brain. Of these 1,437, a total of 987 genes can be mapped to the human interactome. Based on this analysis, 12 of the placebome module proteins (including 7 placebome seed connectors) are brain specific, indicating that the placebome module is significantly enriched with brain-specific proteins $\left(P=6.3 \times 10^{-5}\right.$ and $P=2.7 \times 10^{-4}$, respectively; Figure 2$)$.

As some of the placebome seed proteins are in neurotransmitter pathways, we next analyzed in which pathways the whole placebome module is enriched. To do so, we used a comprehensive database, MSigDB5.1, a collection of annotated gene sets and signaling pathways, for pathway enrichment analysis. The results of this analysis can be found in Supplemental Table 2 (Supplemental File 2), from which we can see that, as expected, some neuro-related pathways are significantly enriched in the placebome module. Importantly, there are also several signaling pathways that are enriched in the placebome module but have not yet been explored regarding the mechanisms of placebo responses, such as the P38/MK2 pathway, PIP3 signaling (in cardiomyocytes), the $\alpha$-synuclein pathway, and the Erbb1 downstream pathway.

Validation of the placebo module using a large cohort of the WGHS. The placebome module we derived from the human interactome based on placebo seed proteins and seed connectors represents potential molecular mechanisms that mediate differences in placebo responses in populations. To validate this point, we used a large population-based cohort from the Women's Genome Health Study (WGHS) (17), a randomized placebo-controlled trial of aspirin and vitamin E for prevention of cardiovascular disease (CVD) and cancer. In this cohort, women were randomly allocated to aspirin or vitamin E, and compared with placebo at 10 years of follow-up. Hall and colleagues have utilized this cohort to examine the effects of the polymorphism (rs4680) of COMT on CVD incidence (17). We considered a subset of the WGHS women allocated to placebo, and used an age-adjusted model $(n=5,814)$ and a fully adjusted model $(n=5,143)$ to examine whether the placebome module contains significantly more genes with SNPs that modify the outcome (total CVD and major CVD incidence) in the placebo arm.

The SNPs in the placebome module genes are compiled from the literature and GWAS catalog (18), jointly maintained by the National Human Genome Research Institute (NHGRI) and the European Bioinformatics Institute (EMBL-EBI). If a placebome module gene had multiple functional SNPs in the 


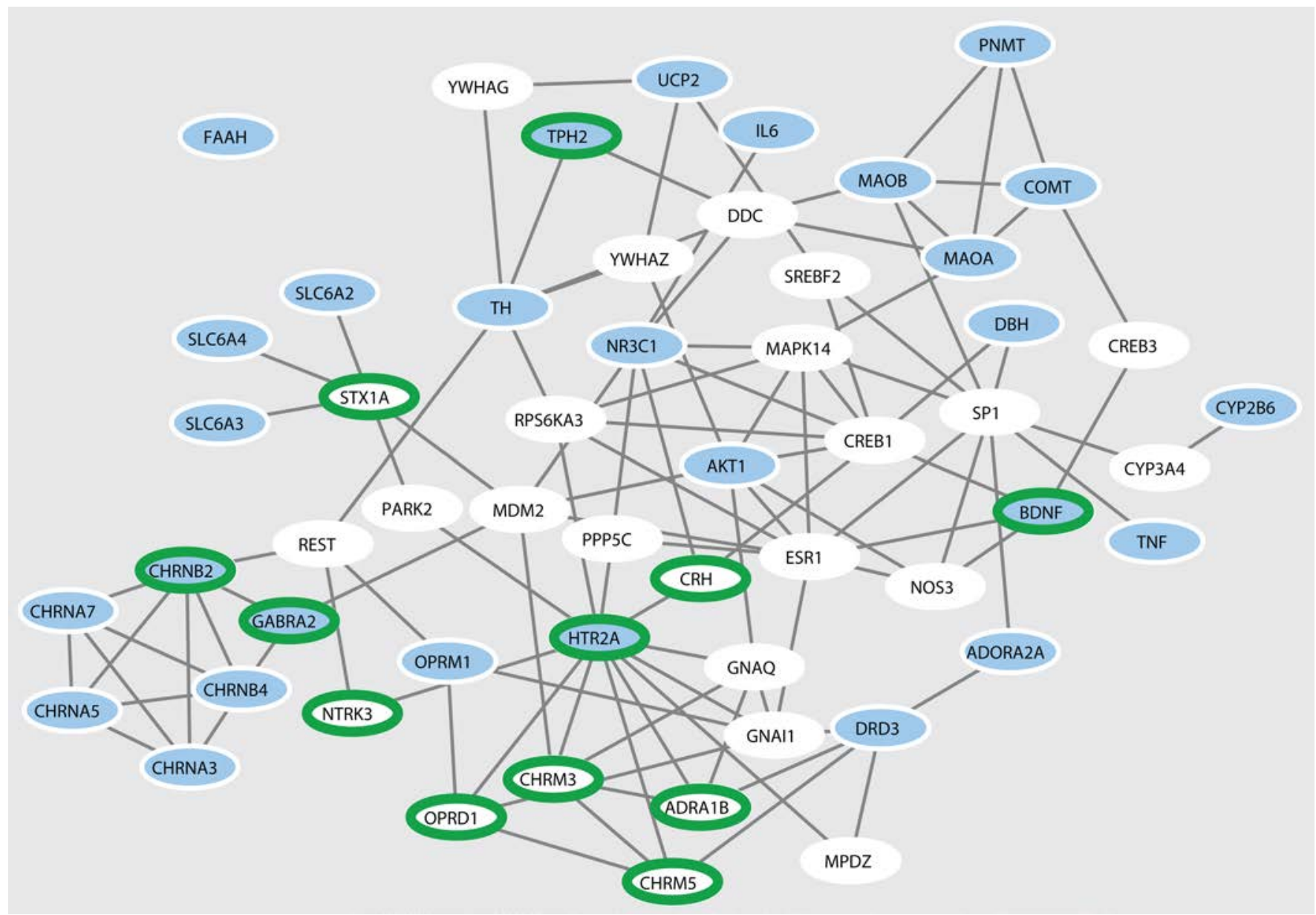

Figure 2. The placebome module. The blue nodes are the placebome seed proteins, and the white nodes are the seed connectors that may also be involved in mediating placebo responses. The nodes with green borders represent (relatively) brain-specific proteins.

GWAS catalog, we selected 1-3 representative SNPs. Because we counted the number of genes that contain significant SNPs (rather than the number of significant SNPs) that modify the outcome of the placebo arm in the enrichment analysis, we did not need to enumerate all of the SNPs in a gene. A list of SNPs we considered in the placebome module and the analytical results can be found in Supplemental Table 3 (Supplemental File 2). Four placebome seed connectors have no functional SNPs from the GWAS catalog or the literature, and thus were excluded in the enrichment analysis. Among the remaining 50 placebome module genes, 17 contain significant SNPs that modify the outcome of the placebo arm (10 are placebome seed genes and 7 are seed connectors, Figure 3). To examine the significance of the enrichment, we collected all of the functional SNPs in the GWAS catalog that satisfy $P$ less than $5.0 \times 10^{-8}$ and pruned them using PLINK (19). This original list of GWAS catalog SNPs $(n=25,226)$ was pruned based on linkage disequilibrium and a variance inflation factor (VIF) of 1.25 , using a window size of 50 SNPs and shifting the window by 5 SNPs at each step. The pruned list of 3,470 GWAS catalog SNPs corresponding to 2,768 genes $(2,077$ of which are represented in the human interactome) was used as our background control. We then randomly selected gene sets of size 50 from the background control 10,000 times and tested how many independent genes contained significant SNPs. The average number of genes that contained significant SNPs from random gene sets was approximately 7.0, indicating that the placebome module was significantly enriched with genes whose SNPs modify the outcome in the placebo arm $(P=8.8 \times$ $10^{-6}$, Supplemental Figure 2 in Supplemental File 1). We also randomly selected gene sets of size 22 (the number of seed connectors) from the background control and found that seed connectors were also significantly enriched with genes that contained significant SNPs $\left(P=5.2 \times 10^{-3}\right.$, Supplemental Figure 2 in Supplemental File 1). Using the 2,077 GWAS genes mapped to the human interactome as the background 


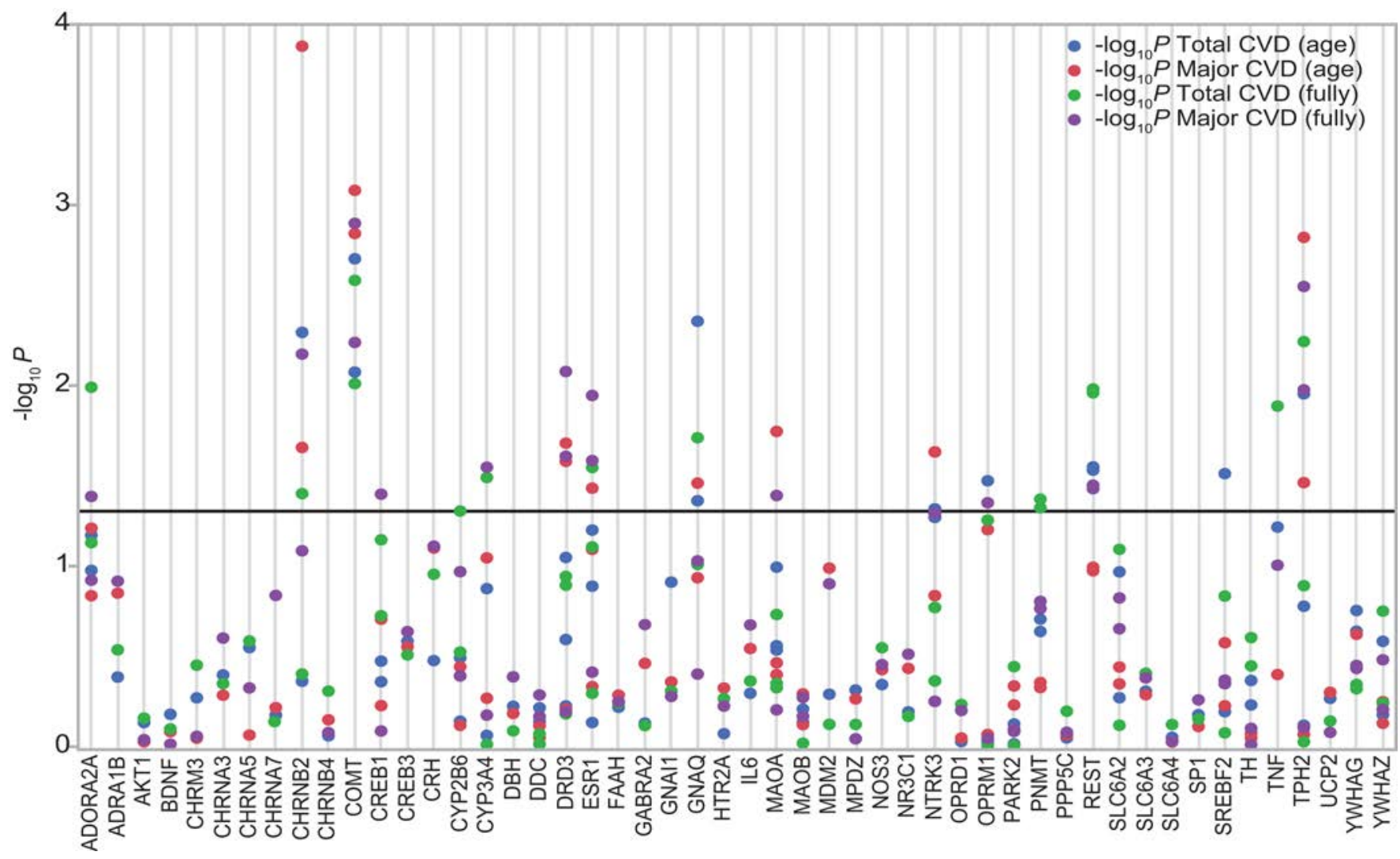

Figure 3. The placebome module genes and significance of their SNPs in modifying the outcome (total cardiovascular disease [CVD] and major CVD incidence) in age- and fully adjusted Cox proportional hazards models of outcomes in the placebo arm of the Women's Genome Health Study (WGHS). Fully adjusted models were adjusted for age, blood pressure, cholesterols, triglycerides, and family history of diabetes mellitus and myocardial infarction.

control led to similar results (Supplemental Figure 2 in Supplemental File 1). Taken together, these results support the hypothesis that the placebome module is a network cluster potentially mediating placebo responses in clinical trials.

Correlation between network proximity and placebo effects. Placebo effects vary widely in different diseases. To assess whether the network proximity of the placebome module to specific disease modules correlates with the strength of placebo effects in corresponding diseases, we collected a list of benchmark diseases in which there was prior knowledge about the placebo response (see Methods). Patient groups with these diseases have been reported to have a range of placebo responses. To conduct a reliable correlation analysis, we only considered meta-analysis studies that cover several clinical trials and also provide a reasonable point estimate for the strength of placebo effects, and excluded those diseases with only single clinical trials even if well designed. In addition to the diseases that have prior knowledge available about placebo effects, we included several negative examples - diseases that have no or low-level placebo responses. These diseases are bacterial infection, infertility, viremia, pneumothorax, and uremia. Placebo effects are known to be minimal in cancer, so we also considered hepatocellular carcinoma and renal cell carcinoma as negative examples given that previous reports show that very low placebo responses were observed in trials of these 2 malignancies (20).

The proximity of the placebome module to the benchmark disease modules in the human interactome is given in Table 1. We note that for the majority of the benchmark diseases that serve as positive examples (i.e., strong placebo responses), the placebome module is significantly closer to their associated modules than random expectation, consistent with prior knowledge about placebo effects in these diseases. For example, in a meta-analysis study considering 27 clinical trials for the treatment of anxiety (and depression), it was reported that the overall (beneficial) change in placebo-treated individuals achieved $79 \%$ (and $76 \%$ ) of the magnitude of the paroxetine response (21). Interestingly, the paroxetine metabolic intermediate, paroxetine catechol, is metabolized by COMT, a node in the placebome (22), 
Table 1. The network proximity between the placebome module and disease modules in the human interactome

\begin{tabular}{|c|c|c|c|c|c|}
\hline \multirow[b]{2}{*}{ Diseases } & \multirow[b]{2}{*}{$\begin{array}{l}\text { Placebo response } \\
\text { (S: strong, W: weak) }\end{array}$} & \multicolumn{2}{|c|}{ Placebome seeds } & \multicolumn{2}{|c|}{ Placebome module } \\
\hline & & Proximity & $\boldsymbol{P}$ & Proximity & $\boldsymbol{P}$ \\
\hline Schizophrenia & $\mathrm{S}$ & 0.11 & $3.4 \times 10^{-22}$ & 0.35 & $2.4 \times 10^{-22}$ \\
\hline Anxiety disorders & 5 & 0.25 & $8.5 \times 10^{-29}$ & 0.54 & $4.2 \times 10^{-27}$ \\
\hline Alcoholism & 5 & 0.29 & $3.5 \times 10^{-26}$ & 0.46 & $1.4 \times 10^{-28}$ \\
\hline Depression & 5 & 0.39 & $1.3 \times 10^{-21}$ & 0.57 & $3.9 \times 10^{-22}$ \\
\hline Parkinson disease & 5 & 0.50 & $7.5 \times 10^{-18}$ & 0.67 & $1.3 \times 10^{-16}$ \\
\hline Eating disorders & 5 & 0.54 & $3.8 \times 10^{-20}$ & 0.65 & $5.7 \times 10^{-26}$ \\
\hline Migraine disorders & $\mathrm{S}$ & 0.79 & $6.8 \times 10^{-18}$ & 0.87 & $1.1 \times 10^{-18}$ \\
\hline Asthma & 5 & 0.96 & $7.3 \times 10^{-7}$ & 0.89 & $1.8 \times 10^{-5}$ \\
\hline Epilepsy & $\mathrm{S}$ & 0.96 & $1.6 \times 10^{-9}$ & 1.04 & $1.2 \times 10^{-8}$ \\
\hline Fibromyalgia & 5 & 1.14 & $2.6 \times 10^{-11}$ & 1.11 & $1.9 \times 10^{-12}$ \\
\hline $\begin{array}{l}\text { Irritable bowel } \\
\text { syndrome }\end{array}$ & $\mathrm{S}$ & 1.11 & $5.3 \times 10^{-9}$ & 1.07 & $4.6 \times 10^{-12}$ \\
\hline Restless leg syndrome & 5 & 1.32 & $1.6 \times 10^{-7}$ & 1.24 & $1.4 \times 10^{-9}$ \\
\hline Diabetic neuropathies & 5 & 1.50 & $2.1 \times 10^{-3}$ & 1.41 & $5.1 \times 10^{-4}$ \\
\hline Crohn's disease & 5 & 1.50 & 0.68 & 1.39 & 0.52 \\
\hline Ulcerative colitis & 5 & 1.68 & 1.00 & 1.48 & 1.00 \\
\hline Duodenal ulcer & 5 & 1.71 & 0.25 & 1.63 & 0.48 \\
\hline Osteoarthritis & 5 & 1.75 & 1.00 & 1.61 & 1.00 \\
\hline Pancreatitis, chronic & $\mathrm{S}$ & 1.79 & 0.67 & 1.78 & 1.00 \\
\hline Infertility & W & 1.25 & $2.6 \times 10^{-3}$ & 1.09 & $1.2 \times 10^{-5}$ \\
\hline Bacterial infections & W & 1.32 & 0.22 & 1.17 & 0.022 \\
\hline $\begin{array}{l}\text { Carcinoma, } \\
\text { hepatocellular }\end{array}$ & W & 1.50 & 0.52 & 1.28 & 0.019 \\
\hline Carcinoma, renal cell & W & 1.68 & 0.46 & 1.44 & $4.8 \times 10^{-3}$ \\
\hline Viremia & W & 1.75 & 1.00 & 1.57 & 0.64 \\
\hline Uremia & W & 2.04 & 1.00 & 2.00 & 1.00 \\
\hline Pneumothorax & W & 2.32 & 1.00 & 2.04 & 0.21 \\
\hline
\end{tabular}

and studies have shown that genetic variation in COMT modifies treatment response to paroxetine in depression (23) and panic disorder (24). The network proximity measure indicates that the placebome module is significantly close to the anxiety module and the depression module in the human interactome. Placebo effects have ranged from $25 \%$ to $75 \%$ in psychopharmacology, and approximately $50 \%$ of recent antipsychotic clinical trials failed to show statistical superiority of the drug tested over placebo, largely owing to the variable placebo response (25-27). In addition, it has been reported that the placebo response is $77.5 \%$ in naltrexone trials for the treatment of alcoholism (7). Our network analysis showed that the schizophrenia module and the alcoholism module were significantly proximate to the placebome module. In contrast, the proximity between the placebome module and most of the negative control disease modules was not significant. These results demonstrate that the proximity measure is a potentially good reflection of the strength of placebo responses. As shown in Table 1, there are certain diseases with known placebo effects whose disease modules are not significantly proximate in the placebome module (e.g., Crohn's disease and ulcerative colitis). This inconsistency may be a reflection of the incompleteness of the interactome or of the different endpoints in the clinical trials. Similarly, the modules of a few diseases with weak placebo effects are statistically proximate to the placebome module (e.g., infertility and renal cell carcinoma), which might be a consequence of the incompleteness of the placebome.

Placebo effects are very heterogeneous, not only among patients with a given disease but also in different clinical studies of the same disease. In addition, numerical definitions of placebo effects are quite different in patient populations with different diseases. For example, in depression, the placebo effect is defined 
A

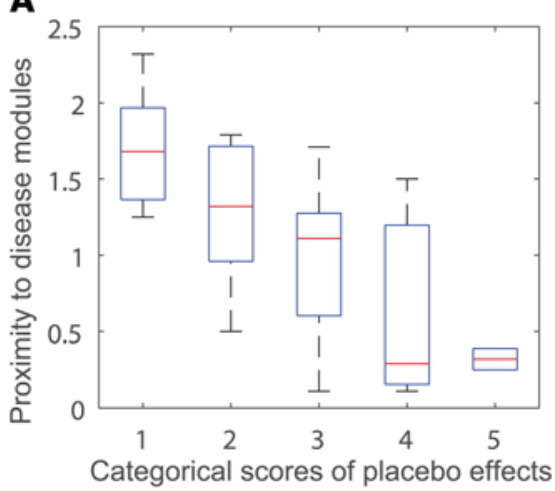

C

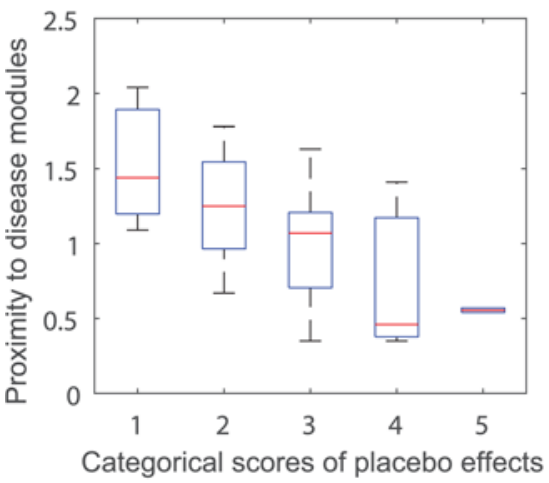

B

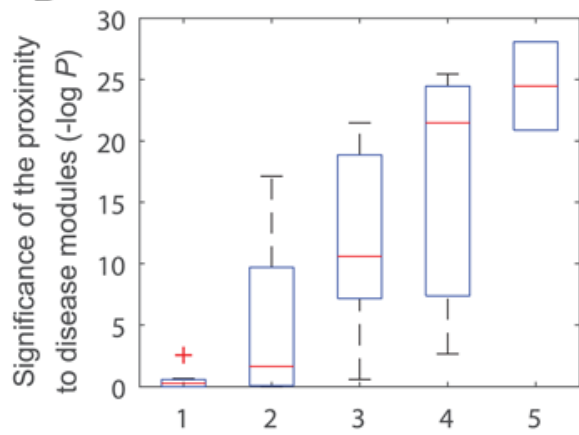

Categorical scores of placebo effects

D

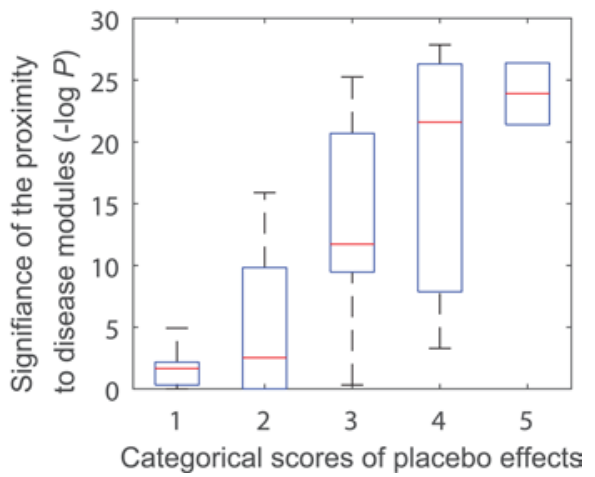

Figure 4. Correlation between the proximity measure and the strength of placebo effects. (A) The proximity of placebo seed proteins to benchmark disease modules in 5 categorical levels of placebo effects. (B) Significance of the proximity between placebo seed proteins and benchmark disease modules in 5 categorical levels of placebo effects. (C) The proximity of the placebome module to benchmark disease modules in 5 categorical levels of placebo effects. (D) Significance of the proximity between the placebome module and benchmark disease modules in 5 categorical levels of placebo effects.

as the percentage of response to antidepressants evaluated by the Hamilton Rating Scale for Depression (HRSD) (28), whereas in alcoholism, the placebo effect is defined as the mean percentage of days abstinent in a placebo group (7). In irritable bowel syndrome (IBS), the frequency of gastrointestinal symptoms in a placebo arm of a clinical trial defines the placebo effect (29). Thus, it is difficult to perform a numerical correlation analysis between network proximity and the strength of placebo effects in different disease populations. Nevertheless, we can assign a categorical score to each disease based on the reported strength of the placebo response in

that disease to unify the variations in placebo effect definitions. Specifically, we assigned a categorical score of 1 for placebo response in the range of $0 \%$ to $10 \%$, regardless of how the placebo response was precisely defined. Similarly, we used scores of 2 for placebo responses in the range of $10 \%$ to $30 \%, 3$ for $30 \%$ to $50 \%$, 4 for $50 \%$ to $70 \%$, and 5 for placebo responses greater than $70 \%$. Five categories were chosen to optimize discrimination among categories, while ensuring that each category has a sufficient number of diseases for robust statistical analysis. For diseases for which there were more than 1 meta-analysis study, we took the average of the strengths of placebo responses. The categorical scores for the benchmark diseases are presented in Supplemental Table 4 (Supplemental File 3).

After we obtained the categorical scores, we calculated the proximity of the modules for these diseases to the placebome module in the interactome. The details for the proximity values can be found in Supplemental Table 5 (Supplemental File 3). We next performed a correlation analysis between network proximity of the disease modules to the placebome module and the strength of placebo effects (Figure 4). We found that the strength of placebo effects in diseases negatively correlates with the proximity of the corresponding disease modules to placebome seed proteins $\left(r=-0.68, P=8.3 \times 10^{-5}\right)$ and positively correlates

Table 2. The network proximity between the placebome module and the symptom modules

\begin{tabular}{|c|c|c|c|c|c|}
\hline \multirow[b]{2}{*}{ Symptoms } & \multirow[b]{2}{*}{ Placebo response (S: strong, W: weak) } & \multicolumn{2}{|c|}{ Placebome seeds } & \multicolumn{2}{|c|}{ Placebome module } \\
\hline & & Proximity & $\boldsymbol{P}$ & Proximity & $\boldsymbol{P}$ \\
\hline Pain & $\mathrm{S}$ & 0.36 & $1.6 \times 10^{-20}$ & 0.54 & $9.2 \times 10^{-22}$ \\
\hline Nausea & $\mathrm{S}$ & 1.11 & $1.8 \times 10^{-11}$ & 1.06 & $1.0 \times 10^{-14}$ \\
\hline Headache & $\mathrm{S}$ & 1.11 & $1.8 \times 10^{-10}$ & 1.04 & $4.4 \times 10^{-14}$ \\
\hline Fatigue & $\mathrm{S}$ & 1.07 & $3.2 \times 10^{-11}$ & 1.06 & $2.7 \times 10^{-12}$ \\
\hline Hot flashes & $\mathrm{S}$ & 1.68 & $6.9 \times 10^{-4}$ & 1.52 & $6.3 \times 10^{-5}$ \\
\hline Fever & W & 1.71 & 1.00 & 1.59 & 1.00 \\
\hline
\end{tabular}

$P$ values were adjusted using the Bonferroni procedure. 


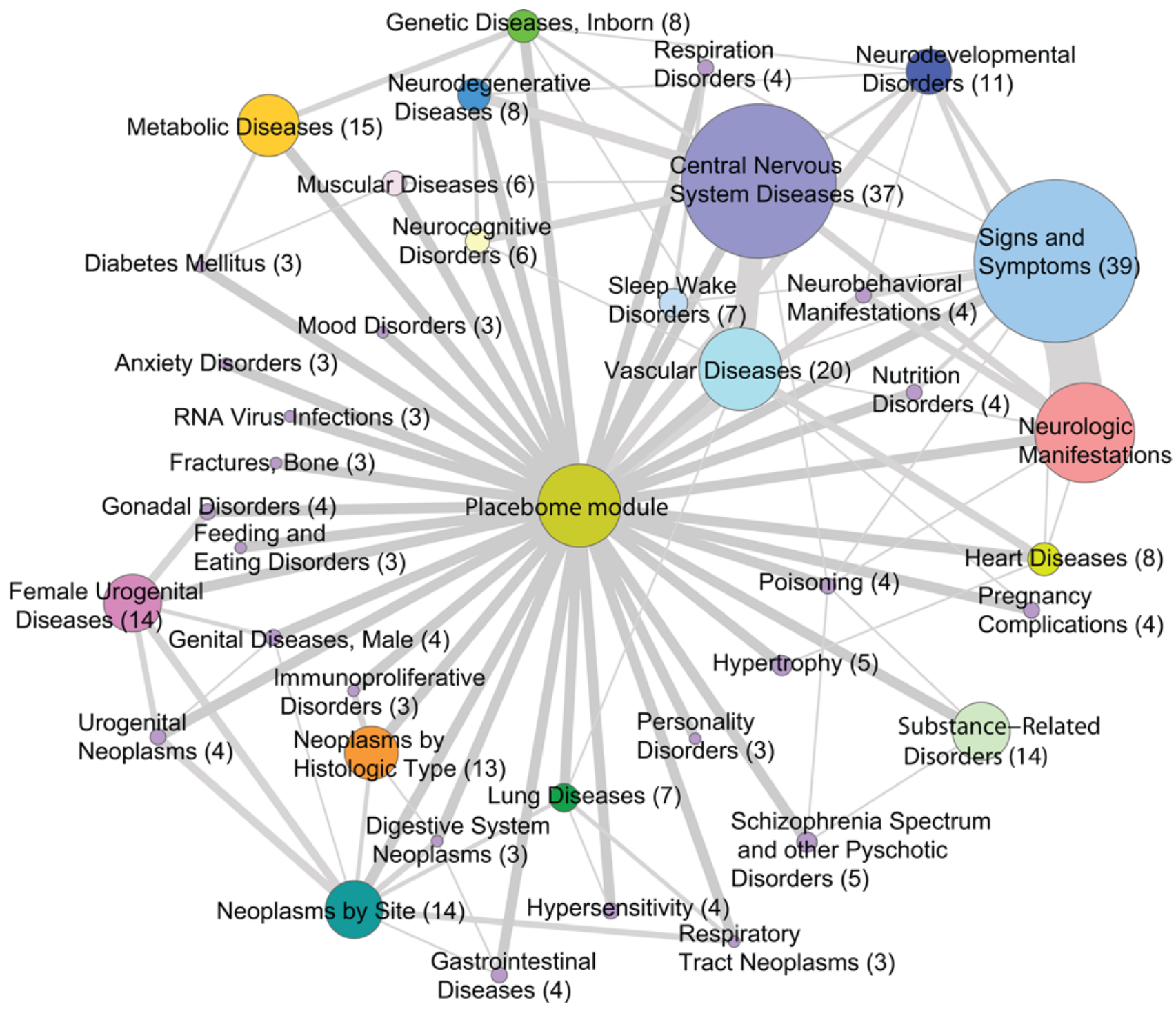

Figure 5. The placebome-disease network. All nodes (except for the placebome module) represent disease groups with the size of the node proportional to the number of diseases in each group. The edges between the placebome module and disease groups represent the significantly proximate relationships of the disease modules in that group to the placebome module. The edges between disease groups indicate that the 2 groups have some shared diseases with the thickness of the edges proportional to the number of diseases in common.

with the significance of the proximity $\left(r=0.76, P=4.8 \times 10^{-6}\right)$. The correlation between the strength of placebo effects in diseases and the proximity of disease modules to the placebome module is also significant (Figure 4, C and D), $r=-0.63, P=4.2 \times 10^{-4}$ and $r=0.74, P=1.2 \times 10^{-5}$ ), confirming the predictive ability of the network proximity measure for placebo effects and the reliability of the placebome module.

In addition to diseases, there are strong placebo responses in patient populations with certain general, common symptoms, such as pain, headache, and nausea. Placebo effects for pain appear to be maximal and increase over time in U.S. clinical trials of neuropathic pain (8). In this symptom analysis, we chose fever as a control symptom since no placebo effect has been observed with this symptom (sign). The proximity of the placebome seeds/module to these symptom modules are summarized in Table 2 . We can see that the placebome module was also proximate to certain pain-related symptom modules. In contrast, the placebo seeds and the placebome module were relatively remote from the fever module. This result further confirms the predictability of the network proximity measure and the reliability of the placebome module.

Proximity of the placebome module to disease modules. The above results suggested that the network proximity measure is predictive of placebo responses in different diseases. Therefore, we next performed a detailed examination of placebo effects in a comprehensive set of 859 diseases (30) by calculating the proximity of 
Table 3. Significance of overlap between the placebome module and drug targets

\begin{tabular}{|c|c|c|c|}
\hline Gene sets & Drug targets & Drug targets in TYPE 1 random gene sets & Drug targets in TYPE 2 random gene sets \\
\hline Placebome seeds & 26 out of 28 & $4.1 \pm 1.9\left(P<1.0 \times 10^{-16}\right)$ & \\
\hline Placebome module & 40 out of 54 & $7.9 \pm 2.6\left(P<1.0 \times 10^{-16}\right)$ & $29.8 \pm 1.8\left(P=8.2 \times 10^{-9}\right)$ \\
\hline
\end{tabular}

the placebome module to their specific interactome-based disease modules. The predicted proximity of disease modules to the placebome module may provide a tool with which to determine the relative magnitude of placebo effects and whether strategies should be implemented to minimize these effects in clinical trials.

The proximity between the placebome and disease modules is reported in Supplemental Table 6 (Supplemental File 4). There were 252 diseases whose interactome-based modules are significantly proximate to the placebome module ( $P<0.05$ after being adjusted by the Bonferroni procedure). These significant diseases can be classified into groups based on their second-level MeSH tree numbers (Figure 5). The constituents of the disease groups are provided in Supplemental Table 7 (Supplemental File 4). Disease groups whose network modules are more proximate to the placebome module include central nervous system diseases (e.g., Parkinson disease, migraine disorders, Alzheimer disease, epilepsy, seizures, Huntington disease), signs and symptoms (e.g., pain, headache, nausea, fatigue, anorexia), and substance-related disorders (e.g., alcoholism, heroin dependence, and tobacco use disorder). Metabolic diseases, vascular diseases, and heart diseases are also proximate to the placebome module, which is consistent with the results of the WGHS analysis above. Some of the diseases proximate to the placebome, such as neoplasm-related diseases, bone fractures, or RNA virus infections, are not clinically known to have objective placebo responses that change pathophysiology (20); the proximity of these diseases suggests that there may be a subset of diseases in which the placebo response can be activated, but lacks efficacy. Thus, activation of a placebo response may not always lead to amelioration of a specific clinical condition. Conversely, in other proximal placebome conditions, such as depression (31) and schizophrenia (32), which are known to have high placebo-related responses, activation of the placebome can be presumed to be efficacious in large subsets of patients. Placebome seed genes like COMT have been associated with myriad diseases, including cancer (33), preeclampsia (34), Parkinson disease (35), CVD (17), and psychiatric disorders (36). The proximity of the placebome to diseases that affect women likely derives from the functional interaction of COMT

Table 4. Drug target categories that are significantly proximate to the placebome module in the human interactome

\begin{tabular}{|c|c|c|c|}
\hline \multirow[b]{2}{*}{ Drug categories } & \multirow[b]{2}{*}{ Size of the targets } & \multicolumn{2}{|c|}{ Placebome module } \\
\hline & & Proximity & $\boldsymbol{P}$ \\
\hline Analgesics, non-narcotic & 142 & 0.96 & $3.5 \times 10^{-10}$ \\
\hline Appetite depressants & 88 & 1.04 & $1.78 \times 10^{-12}$ \\
\hline Antidepressive agents & 262 & 1.04 & $8.6 \times 10^{-5}$ \\
\hline Sympathomimetics & 165 & 1.07 & $2.6 \times 10^{-6}$ \\
\hline Antiparkinson agents & 179 & 1.07 & $6.0 \times 10^{-6}$ \\
\hline Cardiotonic agents & 72 & 1.09 & $1.2 \times 10^{-11}$ \\
\hline Serotonin uptake inhibitors & 140 & 1.11 & $6.5 \times 10^{-7}$ \\
\hline Central nervous system depressants & 78 & 1.13 & $6.1 \times 10^{-9}$ \\
\hline Antioxidants & 116 & 1.19 & $1.4 \times 10^{-5}$ \\
\hline Dopamine agents & 78 & 1.22 & $6.5 \times 10^{-7}$ \\
\hline Excitatory amino acid antagonists & 99 & 1.22 & $1.5 \times 10^{-5}$ \\
\hline Dopamine uptake inhibitors & 74 & 1.30 & $1.7 \times 10^{-5}$ \\
\hline Adrenergic $\alpha$-agonists & 126 & 1.30 & $9.1 \times 10^{-3}$ \\
\hline Neuroprotective agents & 43 & 1.31 & $2.5 \times 10^{-7}$ \\
\hline Adrenergic $\beta$-agonists & 28 & 1.50 & $3.1 \times 10^{-4}$ \\
\hline
\end{tabular}


Table 5. Seed genes in the placebome and their genetic variations associated with placebo responses

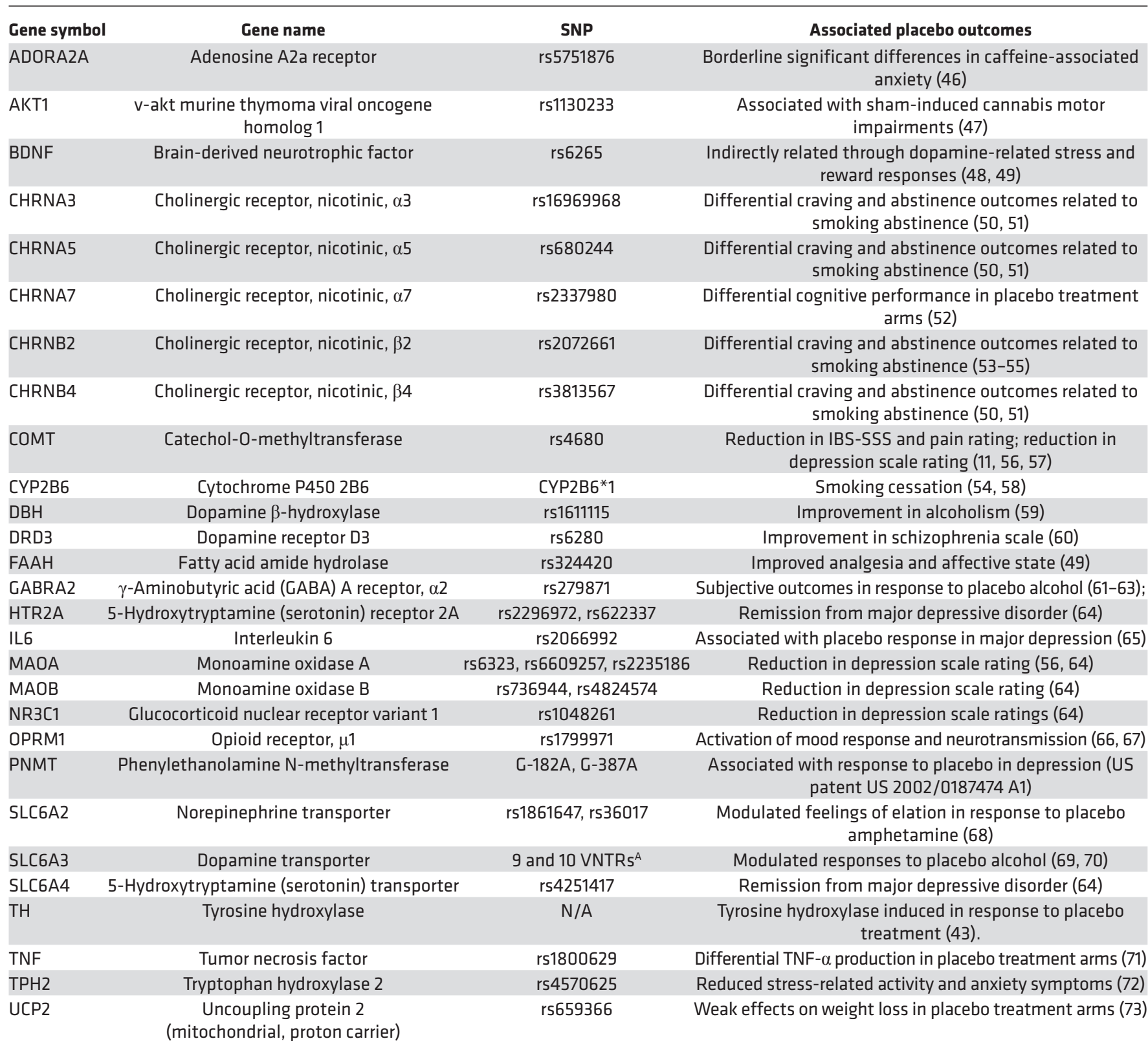

AVNTRs, variable number tandem repeats.

and $M A O-A$ with the sex hormones, estrogen (37) and progesterone $(38,39)$. Thus, it is not surprising that female urogenital diseases and pregnancy complications, which are related to sex hormone levels, are also highly susceptible to placebo responses (40).

Since we have prior knowledge of the placebo effects in some diseases, we can check the groups that contain the benchmark diseases and predict that (non-benchmark) diseases in the same groups likely have strong placebo effects, as well. For example, respiratory disorders and respiratory hypersensitivity are in the same group as asthma, and heroin dependence and other substance abuse-related disorders are in the same group as alcoholism. We predict that these diseases may have strong placebo effects. Sleep disorders and narcolepsy are in the same group as restless leg syndrome, and thus are predicted to have high rates of placebo effects. The results of such a comprehensive list of diseases can provide useful insights into the magnitude of placebo effects in the broad scope of human diseases. 
Proximity of the placebome module to drug targets. Previous studies show that placebo effects also vary across drug treatment trials for the same diseases, suggesting that placebo-drug interactions may exist (41). Knowledge about placebo-drug interactions is important for clinical trial design, as a placebo is commonly used in clinical trials as the direct, comparative arm for the treatment arm. We first ascertained whether the placebome seed genes are themselves drug targets. We downloaded a pool of drug targets from DrugBank (2,095 of which can be mapped to the interactome) (42) and examined the overlap between placebome seed proteins and drug targets. Interestingly, more than $90 \%$ of the placebome proteins are drug targets (Table 3 ). Compared with a protein set that was randomly selected from the interactome (TYPE I random protein set), this percentage was highly significant $\left(P<1.0 \times 10^{-16}\right)$. The majority of the gene products in the placebome module were also drug targets. Thus, the enrichment of drug targets in the placebome module was significant $\left(P<1.0 \times 10^{-16}\right)$. To control bias from the seed proteins in the placebo module, we created a TYPE II random protein set in which the 28 proteins were identical to the seed proteins, but the rest were randomly selected from the human interactome. We found that the placebome module is still significantly enriched with drug targets even with this TYPE II random protein set as the control background $(P=8.2 \times$ $\left.10^{-9}\right)$, indicating that the placebome module is significantly enriched with drug targets.

Lastly, we assessed whether the placebome module is particularly proximate to certain drug categories. To this end, we collected drug categories from DrugBank and calculated the network proximity of the placebome module to the drug targets in each drug category. The results for the full list of drug categories are provided in Supplemental Table 8 (Supplemental File 5). We illustratively selected a few drug categories and provide their proximity to the placebome module in Table 4 . We can see that the placebome module is significantly proximate to a few drug (target) categories; some of the drug categories are used to treat neuro-psychiatric diseases, such as central nervous system depressants, neuroprotective agents, and dopamine uptake inhibitors. In contrast, the placebome module is remote from other drug categories, e.g., sodium chloride symporter inhibitors (Supplemental Table 8), indicating that the placebome module is less likely to cross-talk with signaling pathways perturbed by these drugs, and thus placebo effects might be small in treatments (treatment trials) involving these drugs. Such results provide information that may be used to guide the design of the control arm of clinical trials of specific drug classes, especially among trial subjects taking medications (for other purposes) that may influence a placebo response.

\section{Discussion}

Although the placebo effect has been investigated for many years, efforts to understand the underlying physiology and molecular pathway(s) involved have been limited. With apparently increasing placebo response rates, placebo effects pose interesting and challenging questions to clinical researchers and drug developers. Recent studies have shown that variation in placebo responses among different patients may be due to their genetic context. In this study, we identified a placebome module in the interactome that may mediate the underlying mechanism(s) of placebo effects. We then assessed the relationships between the placebome module and various diseases or drug categories. We found that the placebome module is significantly proximate to the network modules of some diseases (and the targets of some drug categories), and remote from others. The correlation between the network proximity to disease modules and placebo effects provides insight for the design of placebo studies in different diseases. The network proximity measure can be used to predict the strength of placebo responses in patient populations.

We predict that some genes may potentially involve mediating the mechanisms of placebo response since they contain significant genetic variants that change the outcome in the placebo arm of the WGHS study. Genetic variants in placebome genes that enhance (e.g., COMT; see ref. 11) or depress the placebo effect may provide a genomic fingerprint or biomarker for excluding or selecting patients for clinical trials to optimize the likelihood of determining a true drug effect. Our network analysis and results may, therefore, have significant implications for clinical trial design. Based on the proximity of disease modules to the placebome module, investigators may design experimental strategies to minimize the placebo effects in clinical trials by excluding subjects with a high likelihood of a placebo response as defined by a genetic signature (e.g., SNP-based), or by balancing the randomization of genetically predisposed placebo responders across all arms of a trial. For the drug classes whose target modules are close to the placebome module, placebos may not be appropriate controls to test the therapeutic efficacy of the drugs, as the placebo effects may change the outcome of clinical trials. Similarly, our results suggest that there may be diseases whose modules are very proximate to the placebome module that might benefit from drug treatments intentionally 
targeting (activating) placebome pathways. This view has direct implications for our notional definition of a placebo, which for some diseases or symptoms may provide true mechanism-based therapeutic efficacy.

In this study, we used the WGHS cohort to validate the placebome module by examining whether the placebome module has more genes with significant SNPs that modify the CVD outcomes of the placebo arm than a random gene set of similar size. This validation is indirect and not entirely straightforward, as our purpose is to evaluate whether the placebome module has more genes with SNPs that are associated with a placebo response. An ideal validation would require a cohort of patients with and without placebo treatment. However, to our knowledge, there is no such study with more typical placebogenic outcomes available. From Figure 5, we can see that the placebome module is close to vascular diseases and heart diseases, which is supportive of using the WGHS to explore placebo outcomes.

In addition, the placebome module was constructed based on our current knowledge of placebo studies. We would expect that the network module induced by placebome seed genes will topologically change if we remove a few genes with weak evidence from the seed gene set. Similarly, in the future, more placebome seed genes would be added once experimental evidence is available. This increase in seed genes will also affect the topology of the placebome module. However, we wish to emphasize that the location and neighborhood of the placebome module in the comprehensive human interactome will unlikely change very much, as seed genes with high evidence have been included in our study. Therefore, the relationships between the placebome module and diseases/drug targets would also unlikely change significantly.

\section{Methods}

The source of placebome seed genes. We performed a comprehensive review of the scientific literature and collected a group of genes or gene products whose genetic variations may modify the placebo response. First, we searched PubMed by using key words — placebo response, gene, and SNP — and identified $n=$ 42 studies (after excluding nonhuman studies) that had specifically investigated genetic variation and the placebo response. Some studies $(n=6)$ known to us did not appear in the PubMed search, and these were examined for potential genes to be added, including 1 study of the placebo response which demonstrated that tyrosine hydroxylase ( $\mathrm{TH}$ ) was induced in response to placebo treatment (43). We reviewed each article for data identifying specific SNPs that modified outcomes in the placebo arm of a clinical trial. We also included those SNPs that modified outcomes in both the drug and placebo arms. As prior knowledge of the placebome is very limited, we have been as inclusive as possible and considered even those genes with weak evidence. Collectively, we ultimately obtained a list of 28 genes (gene products) as the seed gene pool for the placebome (Table 5). We then mapped their protein products into the human interactome, which is described in the section "Compiling the comprehensive human interactome" in Supplemental File 1.

Connecting the placebome seed proteins. Owing to the incompleteness of placebome seed proteins and of the human interactome itself, these seed proteins may not be densely connected to each other to form a network module. Therefore, we developed an algorithm, called the Seed-Connector algorithm (See the section "Connecting the placebome seed proteins" in Supplemental File 1), in which we attempted to connect the placebome seed proteins by using as few extra nodes as possible. The principle underlying this algorithm is that seed proteins should not be very far from each other, and thus should reach each other through short paths. The placebome module obtained by this algorithm has a very high ratio of seed proteins to connector proteins.

Disease modules and drug categories. To gain insights into placebo responses in different diseases, we collected a list of 20 benchmark diseases (18 known to have moderate or high placebo responses and 2 known to have little or no placebo response) and 5 symptom phenotypes on which we have prior knowledge about the placebo response based on the literature. The benchmark diseases with high responses are alcoholism, anxiety, asthma, Crohn's disease, depression, diabetic neuropathies, duodenal ulcer, epilepsy, eating disorders, fibromyalgia, irritable bowel syndrome, Parkinson disease, migraine disorders, osteoarthritis, chronic pancreatitis, restless leg syndrome, schizophrenia, and ulcerative colitis. The 2 with little or no responses are hepatocellular carcinoma and renal cell carcinoma. The 5 symptoms we considered are pain, headache, nausea, fatigue, and hot flashes. For prediction purposes, a comprehensive list of 859 diseases were obtained from Phenopedia. We obtained their associated genes from Phenopedia in the HuGE navigator (30) and mapped the disease- or symptom-associated gene products to the human interactome and derived disease modules or symptom modules. We also collected 193 drug categories and their targets from DrugBank in which drugs are categorized into different groups based on their therapeutic indications. The 
detailed method for collecting disease genes and drug targets can be found in the section, "Disease modules and drug categories" in Supplemental File 1. We then assessed the relationships between the placebome module and disease modules or drug target modules at the network level.

Network proximity measure. The distance (the length of shortest paths) between 2 proteins or 2 protein sets in a molecular network is often used to characterize their relationships at the systems level. To investigate the placebo effects in different diseases and drug treatments, we used a network measure to calculate the proximity of the placebome module to disease modules and drug target modules. The network proximity measure is defined as the average minimum shortest path length in the interactome from the placebome module to a disease module or a drug target module: $P=\left\langle p_{s}\right\rangle$ and $p_{s}=\min _{d}\left(L_{s d}\right)$, where $p_{\mathrm{s}}$ is the minimum shortest path length in the human interactome from a placebome protein $s$ to the associated proteins of a disease or the targets of a drug category. This measure has been used to explore the proximity between drug targets and disease proteins (44).

Statistical tests and tools. Network visualization was performed with the open source platform Cytoscape (45). When we assessed the topological properties of the placebome module, we created a random control for significance, i.e., we randomly selected a protein set of the same size as the placebome module and calculated the topological properties of the random modules. The significance of the proximity between the placebome module and disease modules or drug target modules was evaluated by creating a random module for each disease or drug target category as a random control. Unless otherwise specified, when we assessed the significance of emergent properties of the observations by comparing them with null models (random controls), all $P$ values were obtained by fitting the histograms to normal distributions using the 'normfit' command in Matlab (Mathworks, Inc). $P$ values (adjusted by the Bonferroni procedure if applicable) less than 0.05 were considered significant. All error bars in the figures are SEM.

\section{Author contributions}

JL and RSW conceived and designed the research. RSW and KTH performed the analyses. FG and DP assisted with the analyses. TK gave constructive suggestions and comments. RSW and JL wrote the manuscript. All the authors revised and approved the manuscript.

\section{Acknowledgments}

This work was supported by NIH grants HL61795, HL108630 (MAPGen Consortium), HL048743, and GM107618 (to JL), and NIH/NCCIH grant 2K24 AT004095 (to TJK). KTH is funded by a Harvard Catalyst faculty fellowship and 1K01HL130625. We wish to thank Albert-Laszlo Barabasi's laboratory at Northeastern University for providing access to the consolidated human interactome data.

Address correspondence to: Joseph Loscalzo, Department of Medicine, Brigham and Women's Hospital, 75 Francis Street, Boston, Massachusetts 02115, USA. Phone: 617.732.6340; E-mail: jloscalzo@partners.org.

1. Kaptchuk TJ. Placebo studies and ritual theory: a comparative analysis of Navajo, acupuncture and biomedical healing. Philos Trans R Soc Lond B Biol Sci. 2011;366(1572):1849-1858.

2. Temple R, Ellenberg SS. Placebo-controlled trials and active-control trials in the evaluation of new treatments. Part 1: ethical and scientific issues. Ann Intern Med. 2000;133(6):455-463.

3. Enck P, Bingel U, Schedlowski M, Rief W. The placebo response in medicine: minimize, maximize or personalize? Nat Rev Drug Discov. 2013;12(3):191-204.

4. Benedetti F, Lanotte M, Lopiano L, Colloca L. When words are painful: unraveling the mechanisms of the nocebo effect. Neuroscience. 2007;147(2):260-271

5. Kaptchuk TJ, Miller FG. Placebo effects in medicine. N Engl J Med. 2015;373(1):8-9.

6. Colagiuri B, Schenk LA, Kessler MD, Dorsey SG, Colloca L. The placebo effect: from concepts to genes. Neuroscience. 2015;307:171-190.

7. Litten RZ, et al. The placebo effect in clinical trials for alcohol dependence: an exploratory analysis of 51 naltrexone and acamprosate studies. Alcohol Clin Exp Res. 2013;37(12):2128-2137.

8. Tuttle AH, et al. Increasing placebo responses over time in U.S. clinical trials of neuropathic pain. Pain. 2015;156(12):2616-2626.

9. Furukawa TA, et al. Placebo response rates in antidepressant trials: a systematic review of published and unpublished doubleblind randomised controlled studies. Lancet Psychiatry. 2016;3(11):1059-1066.

10. Hall KT, Loscalzo J, Kaptchuk TJ. Genetics and the placebo effect: the placebome. Trends Mol Med. 2015;21(5):285-294.

11. Hall KT, et al. Catechol-O-methyltransferase Val158Met polymorphism predicts placebo effect in irritable bowel syndrome. PLoS One. 2012;7(10):e48135.

12. de Souza N. Systems biology: an expanded human interactome. Nat Methods. 2015;12(2):107. 
13. Wang RS, Maron BA, Loscalzo J. Systems medicine: evolution of systems biology from bench to bedside. Wiley Interdiscip Rev Syst Biol Med. 2015;7(4):141-161.

14. Menche J, et al. Disease networks. Uncovering disease-disease relationships through the incomplete interactome. Science. 2015;347(6224):1257601.

15. Pradhan AA, Befort K, Nozaki C, Gavériaux-Ruff C, Kieffer BL. The delta opioid receptor: an evolving target for the treatment of brain disorders. Trends Pharmacol Sci. 2011;32(10):581-590.

16. Uhlen M, et al. Proteomics. Tissue-based map of the human proteome. Science. 2015;347(6220):1260419.

17. Hall KT, et al. Polymorphisms in catechol-O-methyltransferase modify treatment effects of aspirin on risk of cardiovascular disease. Arterioscler Thromb Vasc Biol. 2014;34(9):2160-2167.

18. Welter D, et al. The NHGRI GWAS catalog, a curated resource of SNP-trait associations. Nucleic Acids Res. 2014;42(Database issue):D1001-D1006.

19. Purcell S, et al. PLINK: a tool set for whole-genome association and population-based linkage analyses. Am J Hum Genet. 2007;81(3):559-575.

20. Chvetzoff G, Tannock IF. Placebo effects in oncology. J Natl Cancer Inst. 2003;95(1):19-29.

21. Sugarman MA, Loree AM, Baltes BB, Grekin ER, Kirsch I. The efficacy of paroxetine and placebo in treating anxiety and depression: a meta-analysis of change on the Hamilton Rating Scales. PLoS One. 2014;9(8):e106337.

22. Maurer HH, Bickeboeller-Friedrich J, Kraemer T. Gas chromatographic-mass spectrometric procedures for determination of the catechol-O-methyltransferase (COMT) activity and for detection of unstable catecholic metabolites in human and rat liver preparations after COMT catalyzed in statu nascendi derivatization using S-adenosylmethionine. J Chromatogr B Biomed Sci Appl. 2000;739(2):325-335.

23. Benedetti F, Colombo C, Pirovano A, Marino E, Smeraldi E. The catechol-O-methyltransferase Val(108/158)Met polymorphism affects antidepressant response to paroxetine in a naturalistic setting. Psychopharmacology (Berl). 2009;203(1):155-160.

24. Woo JM, Yoon KS, Choi YH, Oh KS, Lee YS, Yu BH. The association between panic disorder and the L/L genotype of catechol-O-methyltransferase. J Psychiatr Res. 2004;38(4):365-370.

25. Agid O, et al. Meta-regression analysis of placebo response in antipsychotic trials, 1970-2010. Am J Psychiatry. 2013;170(11):1335-1344.

26. Pilla Reddy V, et al. Structural models describing placebo treatment effects in schizophrenia and other neuropsychiatric disorders. Clin Pharmacokinet. 2011;50(7):429-450.

27. Laporte JR, Figueras A. Placebo effects in psychiatry. Lancet. 1994;344(8931):1206-1209.

28. Fournier JC, et al. Antidepressant drug effects and depression severity: a patient-level meta-analysis. JAMA. 2010;303(1):47-53.

29. Patel SM, et al. The placebo effect in irritable bowel syndrome trials: a meta-analysis. Neurogastroenterol Motil. 2005;17(3):332-340.

30. Yu W, Clyne M, Khoury MJ, Gwinn M. Phenopedia and Genopedia: disease-centered and gene-centered views of the evolving knowledge of human genetic associations. Bioinformatics. 2010;26(1):145-146.

31. Kirsch I, Deacon BJ, Huedo-Medina TB, Scoboria A, Moore TJ, Johnson BT. Initial severity and antidepressant benefits: a meta-analysis of data submitted to the Food and Drug Administration. PLoS Med. 2008;5(2):e45.

32. Alphs L, Benedetti F, Fleischhacker WW, Kane JM. Placebo-related effects in clinical trials in schizophrenia: what is driving this phenomenon and what can be done to minimize it? Int J Neuropsychopharmacol. 2012;15(7):1003-1014.

33. Zhou Q, et al. Association between the COMT Val158Met polymorphism and risk of cancer: evidence from 99 case-control studies. Onco Targets Ther. 2015;8:2791-2803.

34. Kanasaki K, et al. Deficiency in catechol-O-methyltransferase and 2-methoxyoestradiol is associated with pre-eclampsia. Nature. 2008;453(7198):1117-1121.

35. Kunugi $\mathrm{H}$, et al. High and low activity alleles of catechol-O-methyltransferase gene: ethnic difference and possible association with Parkinson's disease. Neurosci Lett. 1997;221(2-3):202-204.

36. Wonodi I, Stine OC, Mitchell BD, Buchanan RW, Thaker GK. Association between Val108/158 Met polymorphism of the COMT gene and schizophrenia. Am J Med Genet B Neuropsychiatr Genet. 2003;120B(1):47-50.

37. Dawling S, Roodi N, Mernaugh RL, Wang X, Parl FF. Catechol-O-methyltransferase (COMT)-mediated metabolism of catechol estrogens: comparison of wild-type and variant COMT isoforms. Cancer Res. 2001;61(18):6716-6722.

38. Salama SA, Jamaluddin M, Kumar R, Hassan MH, Al-Hendy A. Progesterone regulates catechol-O-methyl transferase gene expression in breast cancer cells: distinct effect of progesterone receptor isoforms. J Steroid Biochem Mol Biol. 2007;107(3-5):253-261.

39. Fernández-Ruiz JJ, Bukhari AR, Martínez-Arrieta R, Tresguerres JA, Ramos JA. Effects of estrogens and progesterone on the catecholaminergic activity of the adrenal medulla in female rats. Life Sci. 1988;42(9):1019-1028.

40. Carpenter JS, et al. Randomized, double-blind, placebo-controlled crossover trials of venlafaxine for hot flashes after breast cancer. Oncologist. 2007;12(1):124-135

41. Schedlowski M, Enck P, Rief W, Bingel U. Neuro-bio-behavioral mechanisms of placebo and nocebo responses: implications for clinical trials and clinical practice. Pharmacol Rev. 2015;67(3):697-730.

42. Law V, et al. DrugBank 4.0: shedding new light on drug metabolism. Nucleic Acids Res. 2014;42(Database issue):D1091-D1097.

43. Lee IS, Lee B, Park HJ, Olausson H, Enck P, Chae Y. A new animal model of placebo analgesia: involvement of the dopaminergic system in reward learning. Sci Rep. 2015;5:17140.

44. Guney E, Menche J, Vidal M, Barábasi AL. Network-based in silico drug efficacy screening. Nat Commun. 2016;7:10331.

45. Shannon P, et al. Cytoscape: a software environment for integrated models of biomolecular interaction networks. Genome Res. 2003;13(11):2498-2504.

46. Rogers PJ, et al. Association of the anxiogenic and alerting effects of caffeine with ADORA2A and ADORA1 polymorphisms and habitual level of caffeine consumption. Neuropsychopharmacology. 2010;35(9):1973-1983.

47. Bhattacharyya S, et al. Protein kinase B (AKT1) genotype mediates sensitivity to cannabis-induced impairments in psychomotor control. Psychol Med. 2014;44(15):3315-3328.

48. Kang HJ, et al. BDNF val66met polymorphism and depressive disorders in patients with acute coronary syndrome. $J$ Affect Disord. 2016;194:1-8.

49. Peciña M, et al. Valence-specific effects of BDNF Val66Met polymorphism on dopaminergic stress and reward processing in 
humans. J Neurosci. 2014;34(17):5874-5881.

50. Sarginson JE, et al. Response to transdermal selegiline smoking cessation therapy and markers in the $15 \mathrm{q} 24$ chromosomal region. Nicotine Tob Res. 2015;17(9):1126-1133

51. Chen LS, et al. Interplay of genetic risk factors (CHRNA5-CHRNA3-CHRNB4) and cessation treatments in smoking cessation success. Am J Psychiatry. 2012;169(7):735-742.

52. Rigbi A, Yakir A, Sarner-Kanyas K, Pollak Y, Lerer B. Why do young women smoke? VI. A controlled study of nicotine effects on attention: pharmacogenetic interactions. Pharmacogenomics J. 2011;11(1):45-52.

53. Conti DV, et al. Nicotinic acetylcholine receptor beta2 subunit gene implicated in a systems-based candidate gene study of smoking cessation. Hum Mol Genet. 2008;17(18):2834-2848.

54. King DP, et al. Smoking cessation pharmacogenetics: analysis of varenicline and bupropion in placebo-controlled clinical trials. Neuropsychopharmacology. 2012;37(3):641-650.

55. Perkins KA, Lerman C, Mercincavage M, Fonte CA, Briski JL. Nicotinic acetylcholine receptor beta2 subunit (CHRNB2) gene and short-term ability to quit smoking in response to nicotine patch. Cancer Epidemiol Biomarkers Prev. 2009;18(10):2608-2612.

56. Leuchter AF, McCracken JT, Hunter AM, Cook IA, Alpert JE. Monoamine oxidase A and catechol-o-methyltransferase functional polymorphisms and the placebo response in major depressive disorder. J Clin Psychopharmacol. 2009;29(4):372-377.

57. Yu R, Gollub RL, Vangel M, Kaptchuk T, Smoller JW, Kong J. Placebo analgesia and reward processing: integrating genetics, personality, and intrinsic brain activity. Hum Brain Mapp. 2014;35(9):4583-4593.

58. Lee AM, et al. CYP2B6 genotype alters abstinence rates in a bupropion smoking cessation trial. Biol Psychiatry. 2007;62(6):635-641.

59. Arias AJ, Gelernter J, Gueorguieva R, Ralevski E, Petrakis IL. Pharmacogenetics of naltrexone and disulfiram in alcohol dependent, dually diagnosed veterans. Am J Addict. 2014;23(3):288-293.

60. Bhathena A, et al. Association of dopamine-related genetic loci to dopamine D3 receptor antagonist ABT-925 clinical response. Transl Psychiatry. 2013;3:e245.

61. Uhart M, et al. GABRA2 markers moderate the subjective effects of alcohol. Addict Biol. 2013;18(2):357-369

62. Pierucci-Lagha A, et al. GABRA2 alleles moderate the subjective effects of alcohol, which are attenuated by finasteride. Neuropsychopharmacology. 2005;30(6):1193-1203.

63. Kareken DA, et al. A polymorphism in GABRA2 is associated with the medial frontal response to alcohol cues in an fMRI study. Alcohol Clin Exp Res. 2010;34(12):2169-2178.

64. Tiwari AK, Zai CC, Sajeev G, Arenovich T, Müller DJ, Kennedy JL. Analysis of 34 candidate genes in bupropion and placebo remission. Int J Neuropsychopharmacol. 2013;16(4):771-781.

65. Maciukiewicz M, et al. Genetic variation in IL-1 $\beta$, IL-2, IL-6, TSPO and BDNF and response to duloxetine or placebo treatment in major depressive disorder. Pharmacogenomics. 2015;16(17):1919-1929.

66. Peciña M, Love T, Stohler CS, Goldman D, Zubieta JK. Effects of the Mu opioid receptor polymorphism (OPRM1 A118G) on pain regulation, placebo effects and associated personality trait measures. Neuropsychopharmacology. 2015;40(4):957-965.

67. Oslin DW, et al. Naltrexone vs placebo for the treatment of alcohol dependence: a randomized clinical trial. JAMA Psychiatry. 2015;72(5):430-437

68. Dlugos AM, Hamidovic A, Palmer AA, de Wit H. Further evidence of association between amphetamine response and SLC6A2 gene variants. Psychopharmacology (Berl). 2009;206(3):501-511.

69. Anton RF, Voronin KK, Randall PK, Myrick H, Tiffany A. Naltrexone modification of drinking effects in a subacute treatment and bar-lab paradigm: influence of OPRM1 and dopamine transporter (SLC6A3) genes. Alcohol Clin Exp Res. 2012;36(11):2000-2007.

70. Ray LA, Bujarski S, Squeglia LM, Ashenhurst JR, Anton RF. Interactive effects of OPRM1 and DAT1 genetic variation on subjective responses to alcohol. Alcohol Alcohol. 2014;49(3):261-270.

71. Belisle SE, Leka LS, Delgado-Lista J, Jacques PF, Ordovas JM, Meydani SN. Polymorphisms at cytokine genes may determine the effect of vitamin E on cytokine production in the elderly. J Nutr. 2009;139(10):1855-1860.

72. Furmark T, et al. A link between serotonin-related gene polymorphisms, amygdala activity, and placebo-induced relief from social anxiety. J Neurosci. 2008;28(49):13066-13074.

73. Hsiao TJ, Wu LS, Hwang Y, Huang SY, Lin E. Effect of the common -866G/A polymorphism of the uncoupling protein 2 gene on weight loss and body composition under sibutramine therapy in an obese Taiwanese population. Mol Diagn Ther. 2010;14(2):101-106. 